\title{
On measuring the local perceived blur in natural scenes both in the lab and online
}

\author{
Christopher Patrick Taylor ${ }^{\mathrm{a}}$, Peter J. Bex ${ }^{\mathrm{b}}$ \\ ${ }^{a}$ Centre for Integrative Neuroscience and Neurodynamics, Department of Psychology and Clinical Language \\ Sciences, University of Reading, Reading, UK \\ ${ }^{b}$ Department of Psychology, Northeastern University, Boston, MA, USA
}

\begin{abstract}
Natural retinal images contain blur. Image blur varies across the visual field depending on the three dimensional image structure and the observers' point of accommodation. There is currently no consensus in the field on how to quantify local perceived blur in natural images. We developed a biologically-plausible measure of local image blur, local slope, which is computed from the output of a set spatial frequency and orientation band-limited filters. To determine if local slope captured the perception of image blur variation, we used a task in which observers traced regions in natural images that matched the perceived blur of a standard blur. We ran our tracing task both under controlled laboratory conditions with a small number of observers and online using a large sample of Amazon Mechanical Turk Workers. There was good agreement across observes on image regions with matched perceived blur and a correlation between local slopes of blur-matching areas of natural and standard images for data collected in the lab and online. These data indicate that local slope may provide a rapid and simple metric of perceived blur in natural scenes for human observers.
\end{abstract}

Email addresses: c.p.taylor@reading.ac.uk (Christopher Patrick Taylor), p.bex@neu.edu (Peter J. Bex) 


\section{Introduction}

Blur is common in images and in our perception of the everyday world. In addition to playing a key role in image quality assessment (Ciuffreda et al., 2006), bur is a factor in normal emmetropization and abnormal environmental blur can lead to the development of myopia or hyperopia (Walker et al., 1978; Hodos and Kuenzel, 1984). In the human visual system, perceived blur can arise from a diverse set of sources. For example, optical aberrations, changes in pupil size, accommodation, relative depth, and motion can all introduce blur. Vision researchers measure perceived blur using a variety of methods, including, but not limited to, acuity charts (Ravikumar et al., 2011), synthetic step-edges (Watt and Morgan, 1983; Pentland, 1987; Subbarao and Gurumoorthy, 1988; Watson and Ahumada, 2010; McIlhagga and May, 2012; Georgeson et al., 2007), natural (Field et al., 1997; Elder, 1999; Haun and Peli, 2011; Webster et al., 2006) and naturalistic images (Murray and Bex, 2010). The development of a single, sound, valid, biologically plausible, and naturalistic method to quantify perceived blur would be an important contribution and would aid work on image quality assessment, optical correction, and emmetropization (Ciuffreda et al., 2005, 2006; Walker et al., 1978; Hodos and Kuenzel, 1984). This paper introduces a novel measure of perceived blur and tests its usefulness via a tracing task completed by a small number of trained psychophysical observers under controlled laboratory conditions and a large sample of Mechanical Turk workers.

One global measure of perceived blur that has been used with photographs of natural scenes, is the global slope of the circularly-summed Fourier power spectrum (e.g., Field et al., 1997; Tolhurst et al., 1992; Webster et al., 2006). This measure is often referred to as global slope and natural images have a characteristic global slope of approximately $1 / f^{\alpha}$ (Field et al., 1987; van der Schaaf and van Hateren, 1996). As a rule, images that have negative values of $\alpha$ that are much greater than two appear blurry, whereas images with an $\alpha$ of around negative 1.5-2 appear natural, and images with an $\alpha$ of greater than 
negative 1 appear sharpened (the edges in the scene are emphasized). Global slope is a simple, controllable, and intuitive measure of image blur, but global slope does not capture important aspects of blur perception. For example, Figure 1 shows two images that have identical global slopes. Despite the identical global slopes of the two images, it is easy to see that the apparent blur in the two images is not identical. Global slope, or any global image blur measure, will miss the contextual aspect of perceived blur and fail to capture the dependency of perceived blur on the observer's point of focus in the scene. Developing local perceived blur metrics is an active area of research in vision research; computational approaches that can estimate perceived image blur have been described by Elder (1999), Marziliano et al. (2002), Wang and Simoncelli (2003), and Vu et al. (2012), among others. To contribute to this literature, we developed an intuitive local measure of perceived blur that is conceptually similar to global slope, is based biologically-plausible filter responses, but can be computed locally and rapidly at each point in the image.

\section{Local Spectral Slope Computation}

To characterize the amount of blur present in local regions of natural images, we defined a novel measure - local spectral slope. Our measure resembles a suggestion first made by DeValois and DeValois (1988). They suggested that blur at various locations in a scene could be computed by the brain by comparing the relative power at spatial frequencies in a relatively small number sub-bands that ranged from low to high frequencies (See page 292 in DeValois and DeValois, 1988). The local slope of the amplitude spectrum provides an estimate of this relative power across spatial scales at each location in the image.

Local spectral slope is computed by convolving each point in the image, $I_{(x, y)}$, with a bank of first derivative of Gaussian filters, $G$, at $j$ spatial scales $(\sigma)$ and $k$ orientations $(\theta)$.

$$
R(x, y)=\sum_{\sigma=1}^{j} \sum_{\theta=1}^{k} I(x, y) * G_{\sigma_{j}, \theta_{k}}
$$



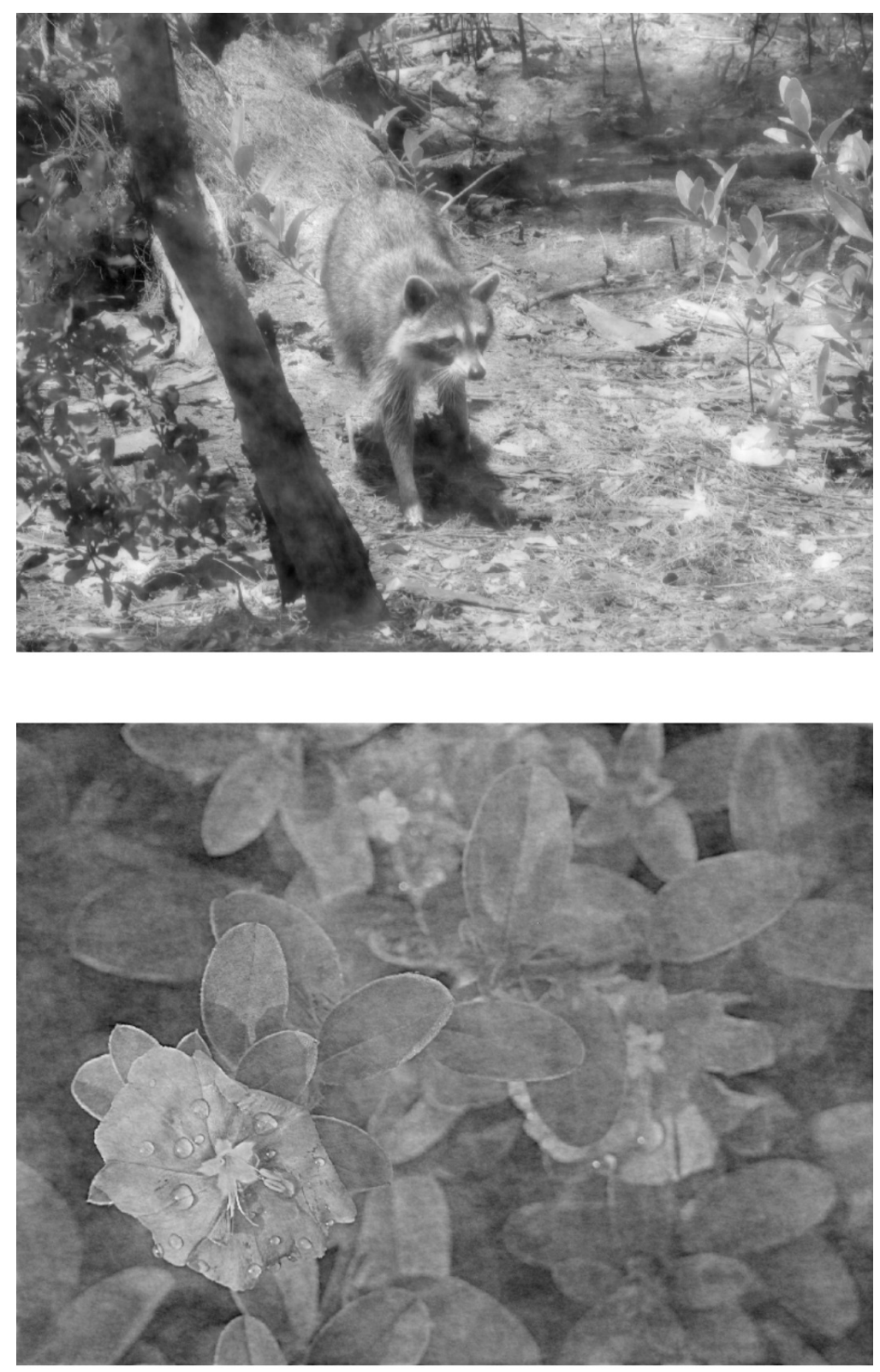

Figure 1: Two images from the database (Olmos and Kingdom, 2004) that have been manipulated to have identical global spectral slope $(\approx-1.39)$ by replacing the original amplitude spectrum of each image with the mean amplitude spectrum of the two images. 
The values in the matrix $R(x, y)$ are the summed filters responses at single position in the image. To arrive a final local slope measure the covariance of $\log ($ frequency) and $\log$ (amplitude) were computed and then normalized by the variance of the filter responses.

To avoid computing slopes at pixels with zero or sub-perceptual threshold contrast, we applied a contrast thresholding operation that has also appeared in other work on perceived blur (Burge and Geisler, 2011; Morgan and Watt, 1997; Georgeson et al., 2007). Our contrast thresholding operation, used a local RMS contrast measure (Mante et al., 2005; Bex et al., 2009) to threshold out low contrast regions in the stimulus, if the local RMS contrast fell below of $5 \%$ then that pixel was excluded from further analysis.

Figure 2 shows a graphical representation of Eq. 1. Part $\mathbf{A}$ of the Figure shows how one region in the original image is converted to a set of local slope measures. The algorithm convolves each pixel in the image on the left with a set of Gaussian derivative filters at several orientations and spatial scales, illustrated in the center. The filters are then steered across orientations (Freeman and Adelson, 1991) to generate an isotropic filter response at each orientation which is then summed. Lastly, response amplitude as a function of spatial frequency is fit by linear regression, depicted by the red line in Figure $2 \mathbf{B}$, to determine the local slope for a given pixel. Once the local slope was computed for all pixels, the result is the image on the right in $\mathbf{A}$. The image on the right in sub-Figure $2 \mathbf{A}$ shows the local slope map that is the result of this computation; in thus image whiter pixels represent shallower local slopes (i.e., sharper regions) which tend to occur at edges in the scene.

\section{Local slope as a measure of perceived blur}

We evaluated our image-based local spectral slope measure of blur with a blur matching task in two observer populations. In order to generate a set of standard images with known blur, we Gaussian low-pass filtered dead leaves images (Lee and Mumford, 2001). Dead leaves images are generated by superimposing large numbers of ellipse elements of variable 

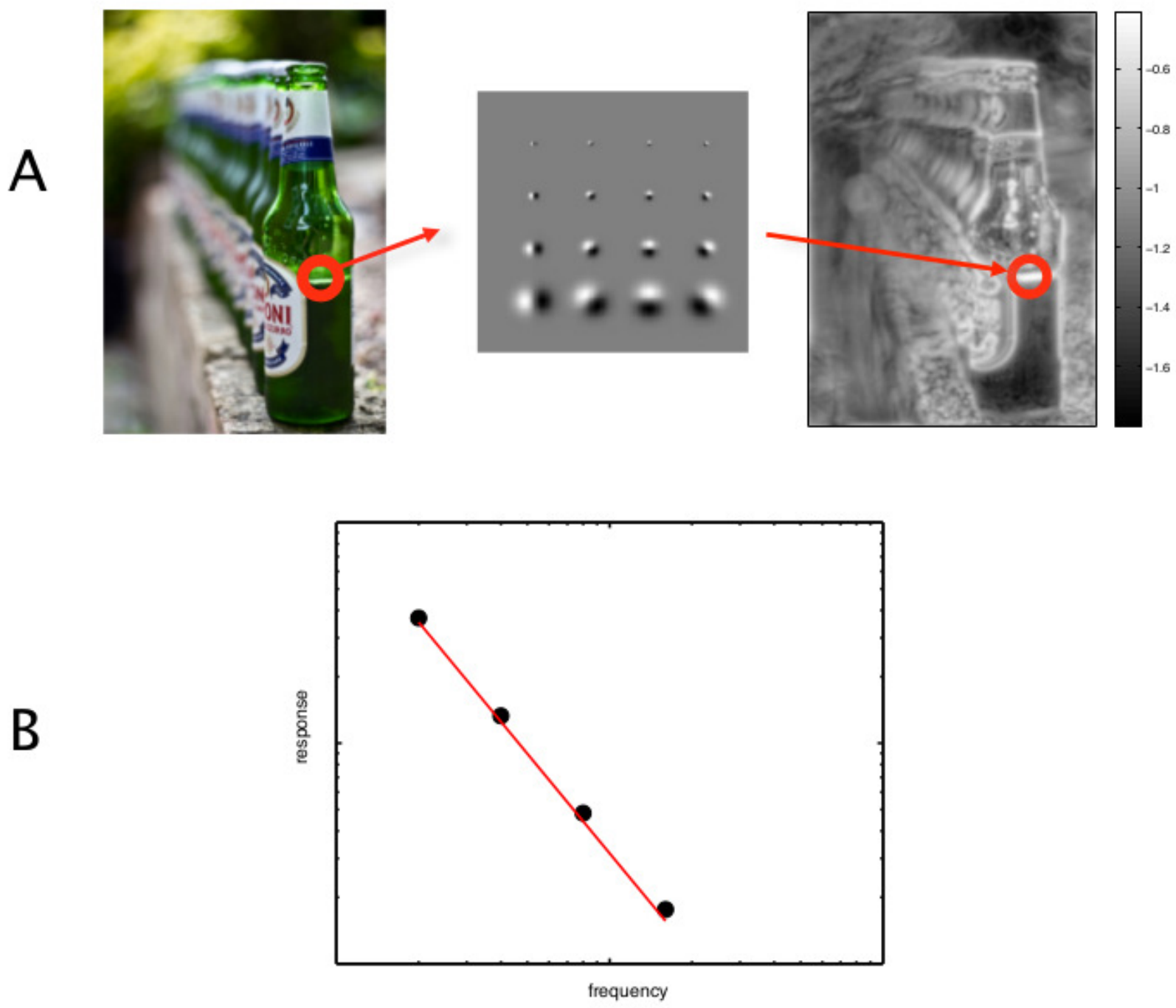

Figure 2: A schematic representation the local slope computation described by Equation 1. A shows how a points in the image are processed by a bank of filters to produce a map of local slopes (the color scale is expressed in $1 / \alpha$ ) and $\mathbf{B}$ shows how individual filter outputs are turned into a single local slope value at each pixel via a correlation of their output. 
luminance, size, aspect ratio and orientation. Dead leaves images share many statistical properties of with natural images, because both contain contours formed by mutual occlusions (Lee and Mumford, 2001), however unlike natural images, the image properties of dead leaves stimuli can be independently controlled. Here, in addition to controlling luminance, size, aspect ratio and orientation, we use a Gaussian low pass filter to control the blur of each element in dead leaves images.

In Experiment 1 psychophysically experienced observers compared the perceived blur in natural images from two databases with the blur of dead leaves patterns of pre-defined blur. Observers traced around regions of natural images in which the perceived blur matched that in the dead leaves standard image. In Experiment 2 Workers from Amazon Mechanical Turk viewed the images from the web and traced regions that matched the perceived blur of a Snellen letter E of pre-defined Gaussian low-pass blur. If local slope is a good measure of

local perceived blur then local slope within the regions that observers trace should correlate with the local slope of the reference stimuli.

\section{Experiment 1: Blur tracing in the lab}

\subsection{Methods}

\subsubsection{Observers}

Four male observers participated in the experiment (including both authors); each observer wore their normal correction and had experience in psychophysical tasks. Their ages ranged from 23-45 and one observer, PJB, is an amblyope. The experiments were approved by and Institutional Internal Review board and carried out in accordance with the Code of Ethics of the World Medical Association (Declaration of Helsinki).

\subsection{Apparatus and stimuli}

Stimuli were presented binocularly to the observers on a Samsung SyncMaster T240HD monitor at a viewing distance of approximately $57 \mathrm{~cm}$. The display was connected to a 
MacBook Pro (2011) running MATLAB and Mac OS X Psychophysics Toolbox 3 (Brainard, 1997; Kleiner et al., 2007; Pelli, 1997). A Spectrascan 655 spectroradiometer (Photo Research Inc) was used to measure luminance to linearize the display. Observers traced their responses with a mouse and the cursor position on screen was recorded as they pressed the mouse button.
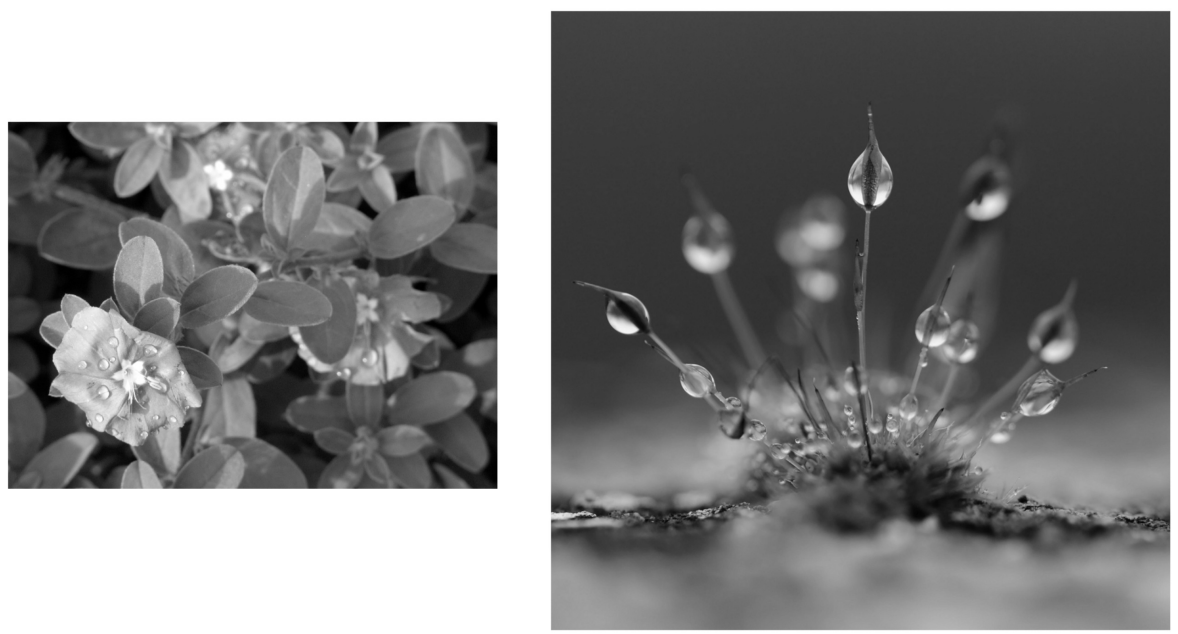

Figure 3: The image on the left is an example of one of the McGill images (Olmos and Kingdom, 2004) used in the blur tracing task. The image on the right is an example of the one of images collected from public domain images from the world wide web. Images are available from the authors.

Two sets of ten gray scale natural images were presented to the observers. The first image set contained ten images selected from the McGill image database (Olmos and Kingdom, 2004). Preliminary work revealed that the images in the McGill image database contained very little blur variation (both subjectively and as measured by our local slope measure). To ensure that the experiment also contained images with a large variation of perceived blur across the images, ta the second set was collected from the web. The web images were selected to be high-resolution (at least 1024 by 768) and were drawn from images in the Creative Commons (Commons, 2001). Figure 3 shows two examples of the images observers 
were asked to trace. All images were scaled-down in size, if necessary, to fit within the 1024 by 768 pixel display area (a visual angle of $\approx 25^{\circ}$ by $17^{\circ}$ ). The mean RMS contrast for both the McGill and Web image sets was 0.49 . The variance of contrast within each set was not controlled - for the McGill images the RMS contrast ranged from 0.43 to 0.72 with a standard deviation of 0.11 and for the Web images the range was 0.32 to 0.67 and standard deviation 0.14 .
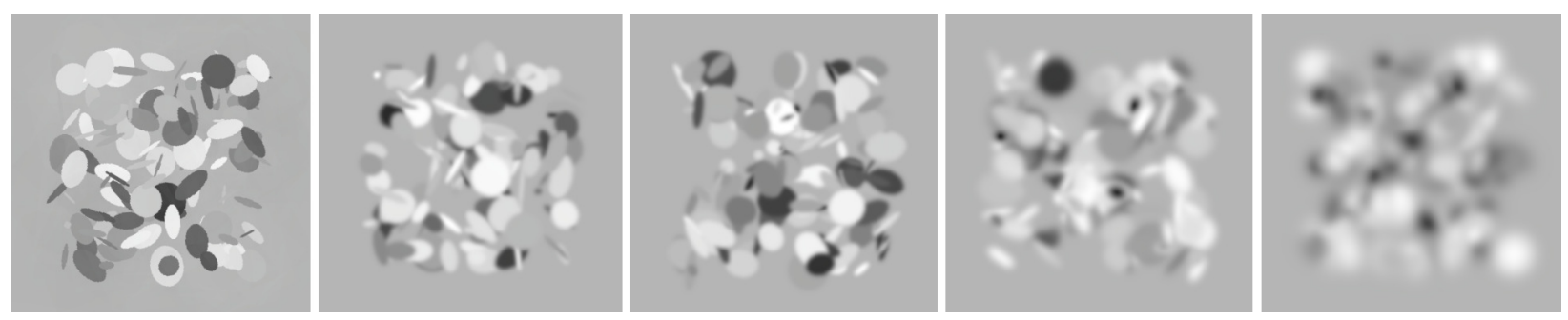

Figure 4: Five examples of the dead leaves stimuli used in the blur tracing task. From left to right the level of Gaussian blur of each element increases from 2 to $64 \mathrm{cy} /$ image. See the text for more detail.

To the right of each natural image, a dead leaves pattern (Bordenave and Gousseau, 2006; Lee and Mumford, 2001) was displayed as a reference stimulus for matching in the tracing task. The dead leaves patterns were constructed from a set of 128 ellipses, and each ellipse was free to vary in aspect ratio, luminance, and orientation. Each ellipse was generated on a local 128 by 128 pixel background and was superimposed in a random position within a 512 by 512 square region on screen. Figure 4 shows examples of the dead leaves stimuli that were used. A Gaussian blur of one of five levels, defined as $\sigma$, was applied to each ellipse element in the dead leaves pattern. The five levels of $\sigma$ were $2,4,8,16,64 \mathrm{cy} /$ image, equivalent to a standard deviation of 90,22, 11, 6 or 3 arcmin, respectively. Examples of patterns with each of the five levels of blur are shown in Figure 4 and the amount of blur present increases in each image from left to right. Stimuli were presented for an unlimited viewing time and observers traced as many regions in the natural image as they wished and were allowed to trace no regions at all if there were no regions in the image that matched the perceived blur 
of the dead leaves.

\subsubsection{Procedure}

Observers were presented with a natural image and a dead leaves reference patch on each trial. The five blur levels of the dead leaves image were randomly interleaved across trials. Observers used the mouse to trace the regions in the natural image that matched the blur of the dead leaves reference stimulus. The experiment was self-paced and observers could obtain additional examples of a dead leaves reference by clicking on the dead leaves pattern. If the pattern was clicked a new random set of ellipses was drawn with the same blur applied to each dead leaves element. Once the observer was satisfied that they had traced all regions in the image that matched the blur of the current dead leaves patch, they clicked a button to begin the next trial. The blur level of the dead leaves reference image on the next trial was selected randomly from those that remained. After all five blur levels had been matched, a new natural image was randomly selected from those that remained in the set for tracing. This procedure was repeated until all twenty test images and five blur match levels had been traced. Examples of individual observers' image tracings are shown in Figure 5.

\subsubsection{Results}

Figure 5 shows four examples of the regions that were traced by four observers when matching $\sigma=64 \mathrm{cy} /$ image (3 arcmin). While there were individual differences in regions observers traced there was broad agreement among the regions observers traced. To estimate agreement among observers for each image/blur level, we computed agreement as:

$$
\kappa=\sum_{x=1}^{w} \sum_{y=1}^{h} T_{x, y} / x y
$$

where the $T_{x, y}$ is a matrix of the sum of the tracings recorded by all the observers at each of the blur levels, normalized by the maximum number of traces at each location. $\kappa$ was then 
Table 1: Agreement $\kappa$ among lab participants.

\begin{tabular}{lcccccccccc}
\hline Image & 1 & 2 & 3 & 4 & 5 & 6 & 7 & 8 & 9 & 10 \\
\hline Web & 0.076 & 0.339 & 0.241 & 0.204 & 0.297 & 0.172 & 0.263 & 0.288 & 0.189 & 0.204 \\
McGill & 0.385 & 0.268 & 0.318 & 0.334 & 0.350 & 0.299 & 0.320 & 0.219 & 0.424 & 0.305 \\
\hline
\end{tabular}

computed by normalizing by the image size, $x y$. This provides a rough measure of agreement across observers for each image. Table 1 provides $\kappa$ for all images in the experiment. In general, Table 1 shows that there was agreement across observers in their tracing behavior for most images. Given that the task was unconstrained and subjective (i.e., observers could trace as much as they wished in the image) the agreement across the images was satisfactory.
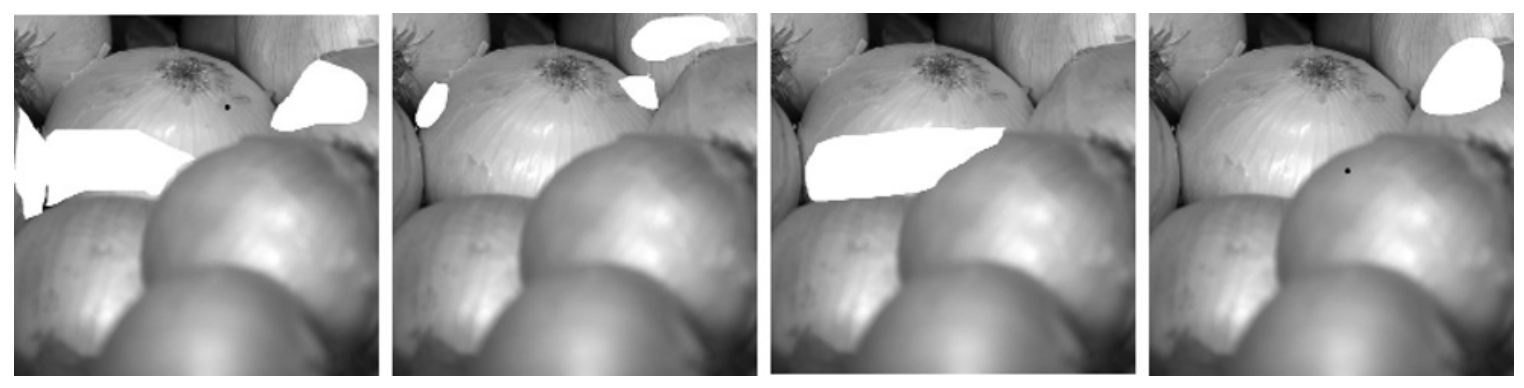

Figure 5: Examples of tracing collected from four observers for an image with one of the reference stimuli with a blur of $64 \mathrm{cy} /$ image ( 3 arcmin) from the Web image set.

Figures 6 and 7 show the correlation between the mean local slope of the dead leaves patch and mean local slope within the polygon traced by the observers. The abscissa was computed by generating a dead leaves patch with a given $\sigma$ (one of $2,4,8,16$, or $64 \mathrm{cy} /$ image) and generating a large set $(1,000)$ dead leaves patches with that level of blur. Local slope was calculated for each of the one-thousand patterns and the mean local slope of the patterns used as the estimated local slope for that blur level. Each point in Fig. 6 and 7 was calculated by averaging the local slope in the traced regions for all images (within each of the two sets) at that blur level. Error-bars in Figs 6 and 7 are 95\% confidence intervals for all tracings in all images within that set for that observer. Each facet of the two Figures contains a dashed 


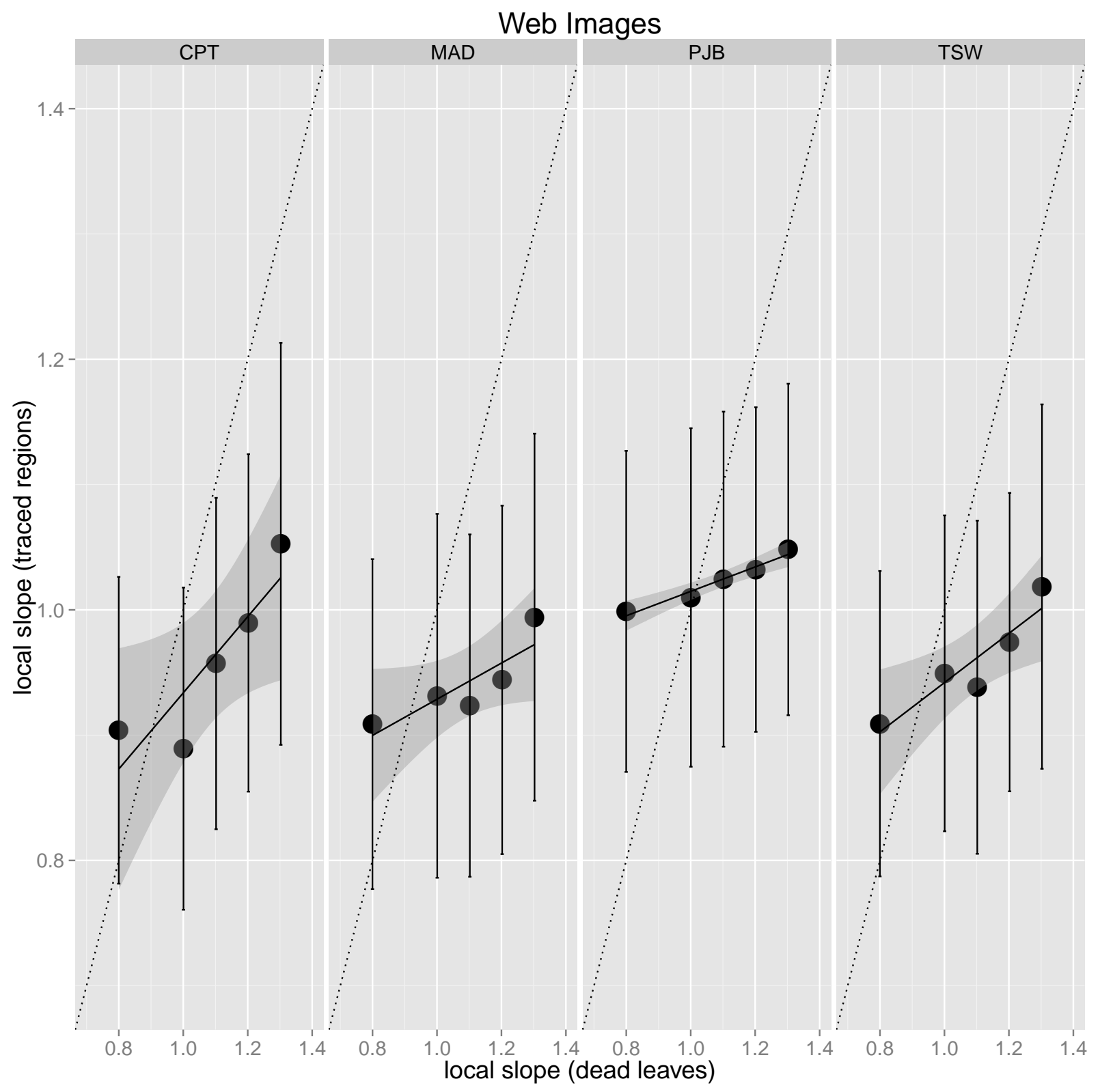

Figure 6: Observers Mean and 95\% confidence intervals for the tracing for four observers. 


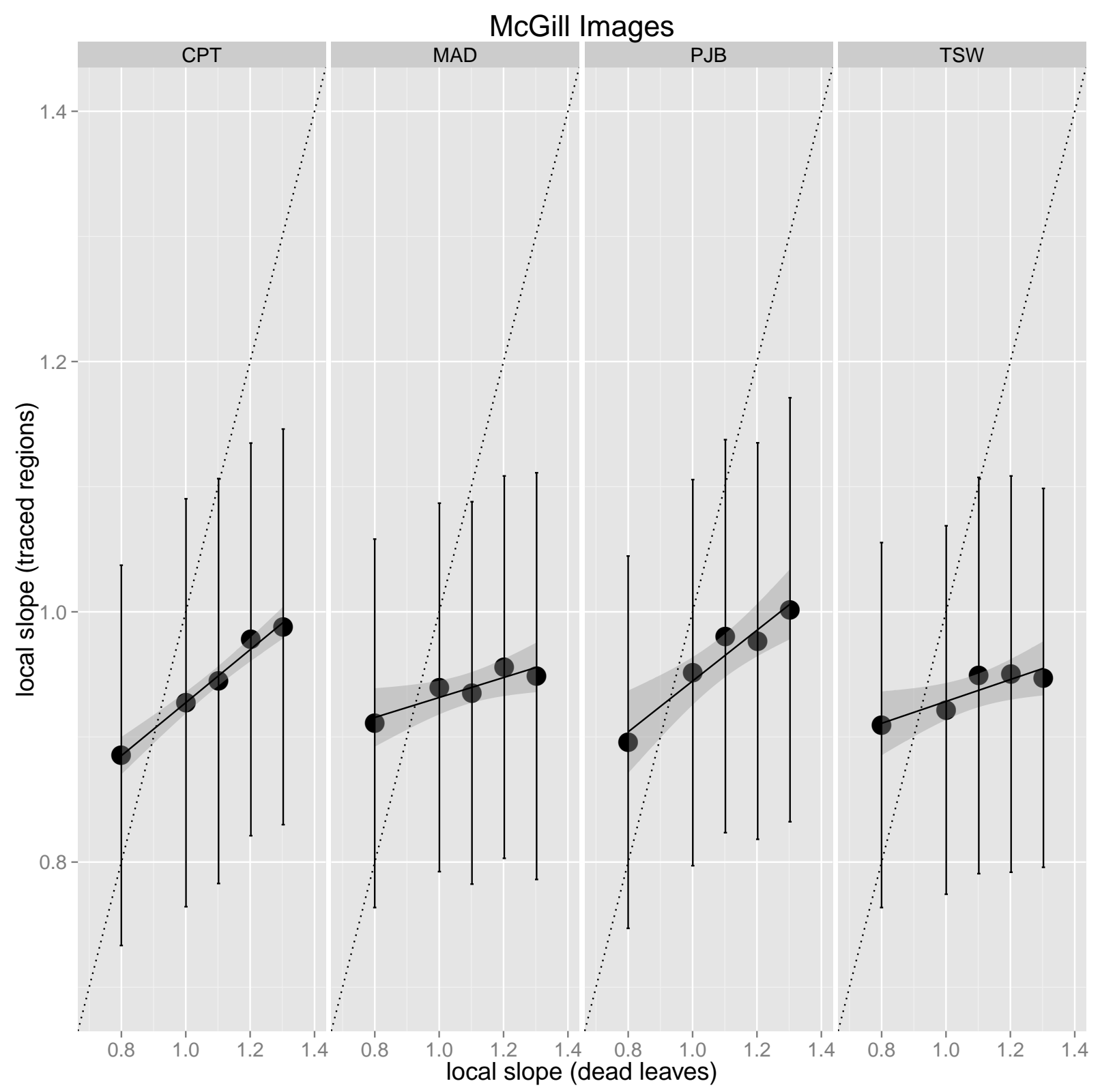

Figure 7: Mean and 95\% confidence intervals for the tracing for four observers. 
Table 2: $\mathrm{R}^{2}$ values for the local slope tracing experiment. ${ }^{*}, p<0.5,{ }^{* *}, p<p<0.01$

\begin{tabular}{lll}
\hline Observer & Web & McGill \\
\hline CPT & $0.785^{*}$ & $0.987^{* *}$ \\
PJB & $0.96^{* *}$ & $0.812^{* *}$ \\
TSW & $0.723^{*}$ & 0.813 \\
MCD & 0.733 & $0.811^{*}$ \\
\hline
\end{tabular}

line at unit slope $(x=y)$. If the data in the plots fell along the unit slope line, then the observer's perceived blur matches would be veridical according to our local slope measure. Table 2 shows summary of correlations, overall there was a good correlation between our local slope measure, but our blur perception matches were not veridical, for all observers fits were shallower than the veridical line. Figs 6 and 7 show linear fits (solid black lines) and confidence regions (gray regions) computed using R.

\section{Experiment 2: blur tracing on Mechanical Turk}

The blur tracing method has at least four disadvantages, it is not bias free, it is a subjective task with no correct answer, data were collected on experienced psychophysical observers who had detailed knowledge of image blur, and observers tracing was not constrained. To address whether our measure of local perceived blur could capture blur perception in a large sample of naive observers, we used Amazon Mechanical Turk to collect blur tracings. The use of online observers loses some control over the apparatus and stimuli by deploying the experiment across a variety of browsers, computers, and monitors. Nevertheless, recent research has found that he data collected on Amazon Mechanical Turk can be of comparable quality and replicate results found in a more controlled laboratory setting (Buhrmester et al., 2011; Crump et al., 2013). Furthermore, many of the images that are used when evaluating image quality or satisfaction with clinical outcomes are viewed on computer or television displays that are not controlled across settings. 


\subsection{Methods}

\subsubsection{Observers}

Observers were recruited via the Amazon Mechanical Turk system and clicked to indicate their consent. A sample of 100 observers traced images for approximately 10 minutes for \$0.5 USD. Five were excluded because they either did not complete the image tracing task or provide demographic information. The median age of the observers was 32 years old with a median absolute deviation of 7 years. The demographics of the sample were: forty-one male observes, fifty-four female, primarily from India $(\mathrm{n}=55)$ and the USA $(\mathrm{n}=30)$, consistent with those reported in previous Mechanical Turk experiments (Ipeirotis, 2010; Ross et al., 2010). India and the USA are over-represented because they are the only two nations in which workers are permitted to withdraw their earnings in cash rather than receive credit with Amazon. The browser type, computer, monitor, and approximate viewing distance were reported by each observer after they completed the experiment.

\subsubsection{Apparatus and stimuli}

JavaScript code originally developed for a online person tracing task (Maji, 2011) was extended and modified with jQuery libraries (McCormick and De Volder, 2004) and customized for use with the Amazon Mechanical Turk worker/requester system. All observers reported using LCD display connected to either a laptop or desktop computer. Not all observers included a monitor measurement in their description, but of those that did the majority reported using 17 inch or 19 inch monitors (at the extremes one reported a 13 inch monitor and another a 24 inch monitor). The mean estimate of viewing distance provided by the observers was $49 \mathrm{~cm}$ with a standard deviation of $12 \mathrm{~cm}$.

Nine of the images from the web set of the previous experiment were used (one image was not included because of a technical error). All the images were gray scale and 450 by 450 pixels in size. The dead leaves patterns were replaced with a Snellen E as a reference 

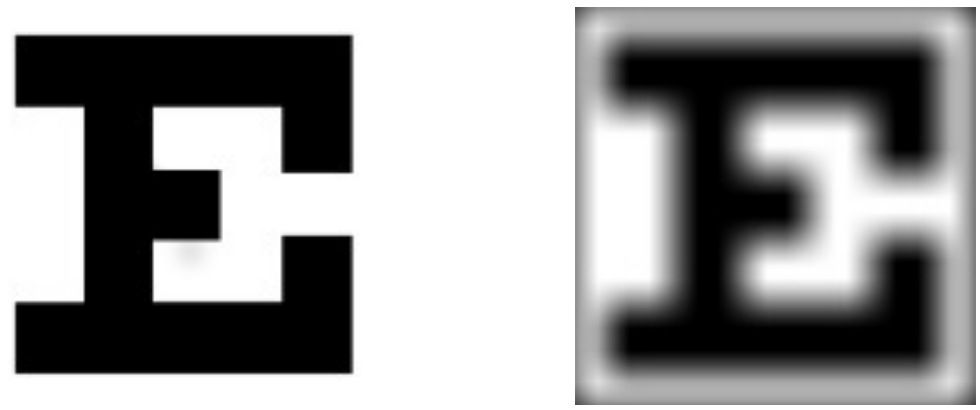

Figure 8: Examples of the Snellen E used as reference stimuli in Experiment 2.

to simplify the task for observers inexperienced psychophysical tasks. Examples of the two Snellen E reference stimuli are shown in Figure 8. The blurred E was created by convolving the Snellen E with a two-dimensional Gaussian kernel with a standard deviation of 8 pixels. The kernel was selected such that the local slope of the blurred E was within the range of local slopes tested in Experiment 1.

\subsubsection{Procedure}

The tracing task was similar to the tracing task used in the lab, but instead of freely tracing curves, observers were instructed to mark out vertices of a polygon that surrounded the region that matched the blur level. The observers were instructed to "Draw the outer boundary of the region in the image that matches the sharpness or bluriness of the E above each image as accurately as possible." Observers viewed four example images (that were not used in the experiment) that showed tracings completed by a volunteer participant naive to the experimental hypotheses. At any point during the experiment, observers could restart their tracing by pressing ' $r$ ' to clear their marked vertices. Once they were finished tracing blur-matching regions in each image, the observer then clicked a button to begin tracing the next image. Observers were allowed unlimited time to do the task but all observers completed the tracing of all 18 images in under 15 minutes. All the code and images for running the experiment are available from the first author's github repository. 
Table 3: Tracing agreement $\kappa$ among Mechanical Turk workers.

\begin{tabular}{lccccccccc}
\hline Image & 1 & 2 & 3 & 4 & 5 & 6 & 7 & 8 & 9 \\
\hline un-blurred & 0.0821 & 0.2457 & 0.1591 & 0.1902 & 0.1606 & 0.1245 & 0.0918 & 0.1089 & 0.1866 \\
blurred & 0.1489 & 0.2902 & 0.1144 & 0.1255 & 0.3452 & 0.1518 & 0.2338 & 0.2182 & 0.2772 \\
\hline
\end{tabular}

\subsubsection{Results}

Figure 9 shows two natural images an example tracing matrices. The tracing matrices (center and right columns) show blur matching areas computed from the sum of all traced polygons ( $T_{x, y}$ from Equation 2) combined across all observers. The two tracing conditions are shown with the original images on the left, tracings for the un-blurred reference in the center, and the blurred reference on the right. There was good agreement within each image of the tracings completed by workers and observers traced a variety of non-object regions (e.g., the blurred tracing in the bottom right of Fig. 9), object parts (e.g., tracings in the top row), and object wholes (the center tracing in the bottom row of Fig. 9). To quantify the agreement among observers we computed $\kappa$ from Equation 2. Table 3 contains the values of $\kappa$ for the 9 blurred and un-blurred images and was computed as in Eq.2. The range of $\kappa$ values were broadly consistent with Experiment 1 and seven of the nine stimuli showed larger agreement for blurred than un-blurred images.

Figures 6 and 7 show tracing data fitted by a linear model. Recall that the slope of the relationship between local blur in the dead leaves images and the local slope in blur matching areas of natural scenes was less than one for all observers. To investigate this finding further we calculated mean local slope of all image tracings for each observer as shown in Figure 10. The correlation between standard local slope and tracing local slope was measure for each pair of images for all observers $\left(r^{2}=0.7174\right)$.

Amazon Mechanical Turk has been shown to produce experimental data that is in good agreement with tasks completed under laboratory conditions across a wide array of cognitive psychology tasks (Buhrmester et al., 2011; Crump et al., 2013) and has been used exten- 


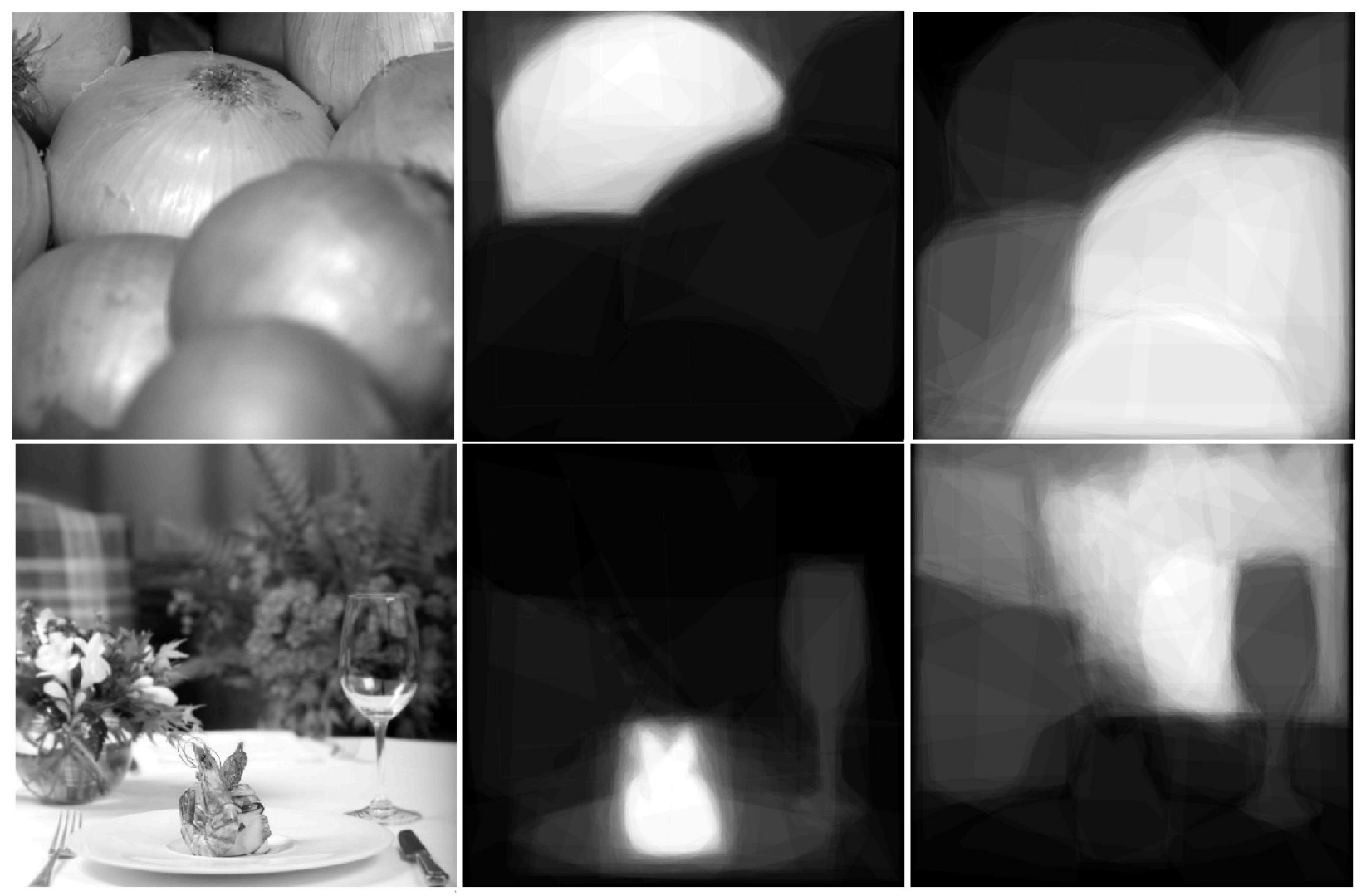

Figure 9: Representative examples of group tracings for the blurred and un-blurred reference stimuli. The white/dark level of the pixel represents the proportion of observers that traced a given region. White in the image indicates pixels traced by the largest number of observers and black or dark gray those regions not traced by any observers. 


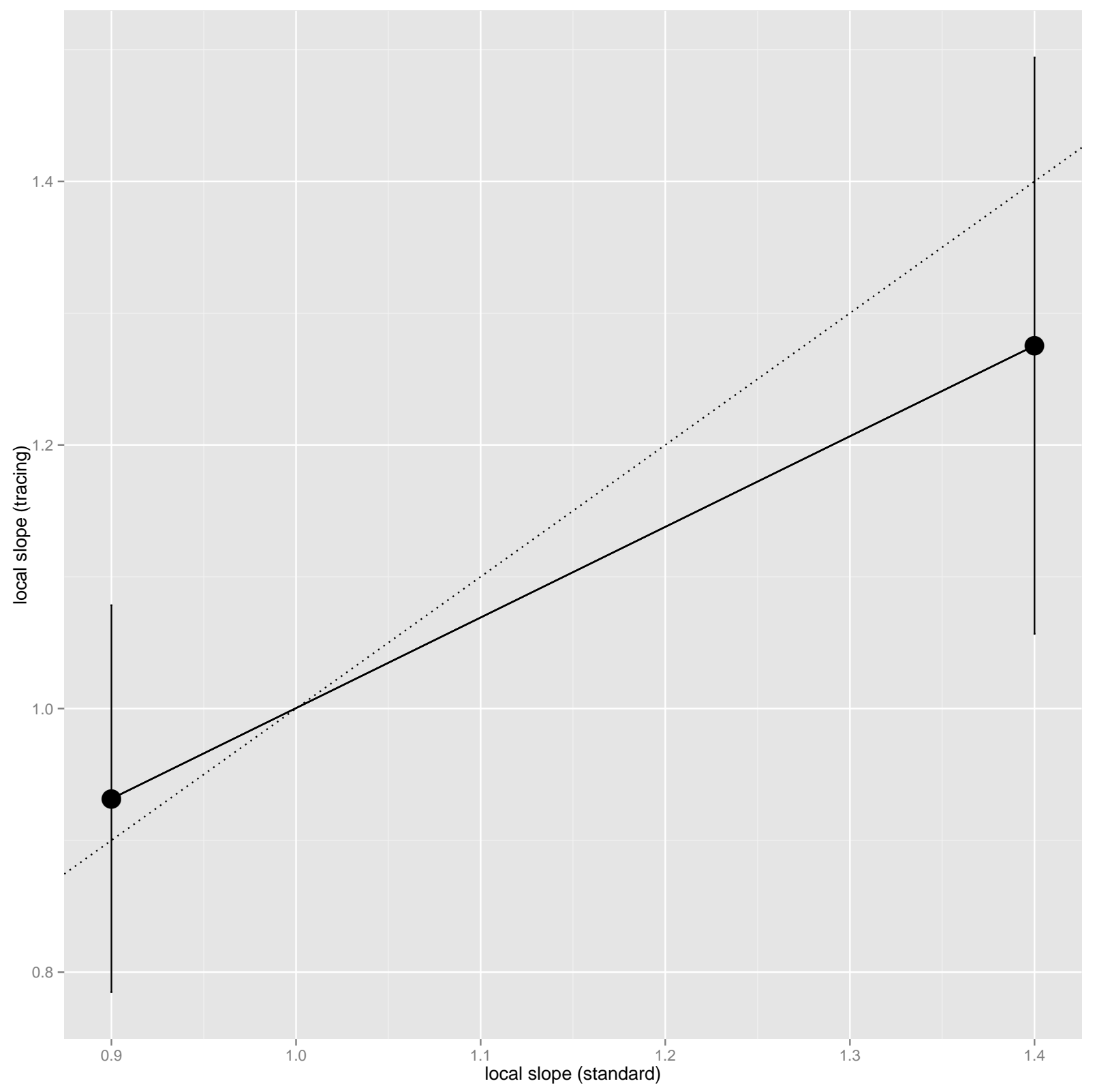

Figure 10: Local slope of the standard versus the reference plotted for all observers for Experiment 2. Error-bars are $95 \%$ confidence intervals. 
sively to collect image annotations both via tracing and verbal report (Sorokin and Forsyth, 2008; Rashtchian et al., 2010; Saunders et al., 2013). However, the lack of stimulus control (e.g., monitor characteristics and viewing distance) is a reason to treat its ability to address perceptual questions with some reservation. Thus, while our correlation between reference and traced blur is within the range observed in the lab, it is important to be cautious when interpreting the results and drawing inferences. Experiment 2 is best considered to be a proof-of-concept that perceived blur may be one area of vision research that may lend itself to online data collection.

\section{Discussion}

In two experiments, experienced and naive observers traced regions of local perceived blur in natural images that matched the blur of a standard stimulus. Both in the lab and on Mechanical Turk there was substantial agreement among observers on the image regions that appeared to have equal levels of image blur. We computed the local slope for the regions traced within of each image and found a significant positive correlation between the local slopes of images with blur of two references image, dead leaves and Snellen letters. In sum, our simple local slope estimate is correlated with perceived blur matches across many observers and can therefore be used as a rapid and easy to compute yet general metric of the distribution of local perceived blur of natural images.

The slope of the matching functions in the lab were shallower than unit slope (see Figs. 6, 7, and 10). Thus the blurry regions in the images appeared less blurred than a similar region of a dead leaves pattern, while a sharp region of a natural scene appeared less sharp that a similar region of a dead leaves pattern. This observation suggests that the perceived range of blur levels in complex images with a broad distribution of blur levels may be higher than the range predicted from simpler images with fewer blur levels.

The perceived blur of synthetic and natural images is known to depend on blur in the 
surround (Webster et al., 2001). The most blurred regions in natural images are necessarily surrounded by sharper image regions and the sharpest regions surrounded by blurrier image regions. These contextual biases would tend to exaggerate the range of blur levels in natural scenes. The dead leaves images contain no such contextual effects because blur is independently applied to each element in the pattern and there is no variation in blur across the image.

\subsection{Relation to other local blur metrics?}

Three examples that quantify perceived image blur have been described by Elder (1999), Wang and Simoncelli (2003), and Vu et al. (2012) - each metric has its benefits and disadvantages computationally. We suggest that the main advantage of the perceived blur metric presented here is its simplicity and intuitive appeal based on previous research on blur and global slope plus its direct relationship to the known properties of receptive fields of neurons in primary visual cortex (DeValois and DeValois, 1988). Because of its simplicity, the model will fail to capture many interesting findings within the perceived blur literature. Any future work that attempts to build on this approach to capture findings in perceived blur will benefit from our metricś minimalism. The simplicity of local slope provides a convenient starting point for adding complexity. For example a weighting of a local blur context, analogous to to local weightings used in work on perceived contrast Haun and Peli (e.g., 2011), may account for contextual effects of perceived blur. One complexity that does not need to be added, the summation of information across spatial frequency and orientation across channels, is present in the local slope calculation. Recall that our local slope measure of perceived blur sums information across spatial frequency and orientation; this summation is consistent with evidence for the summation of spatial frequency and orientation information (Olzak and Thomas, 1999; Taylor et al., 2009, 2014). 


\subsection{Sharpness constancy}

It has been observed that the perceived blur of edges that are drifting at high speed (Bex et al., 1995), viewed with peripheral visual field (Galvin et al., 1997), or viewed with

an impaired visual system (Simmers et al., 2003) are often sharper than expected by the spatial resolution of the visual system. This phenomenon has been attributed to gain control mechanisms (Georgeson, 1985) or to prior knowledge learned by the visual system (Burr and Morgan, 1997). Our local slope measure would be unaffected by the absence of spatial filters at high spatial frequencies in any of the conditions described above. Thus, if the visual system computes a quantity analogous to local slope then we may have a simple measure of sharpness phenomena that does not require additional mechanisms or model complexity beyond what we have presented.

\subsection{Blur and depth perception}

The role of blur in depth perception has been considered by researchers for some time (Pentland, 1987; Ens and Lawrence, 1993; Mather, 1997; Mather and Smith, 2000a,b, 2002). Recent advances in display technology and photography, (e.g., Watt et al., 2005; Burge and Geisler, 2011; Held et al., 2012) have enabled researchers to explore how blur is involved in the computation of depth under naturalistic conditions. The lack of depth in our model is a limitation of our measure - blur and depth of focus are intimately related when viewing real scenes in the world. As discussed above, local context cues can alter perceived blur and if the visual system were able to segment surfaces in depth, then some of the contextual blur effects may be eliminated or minimized. Extending our local slope measure to include a role for depth, would bring together the research on blur as a depth cue and our work on perceived blur. For example, local slope could be calculated at a small number of multiple depth planes in the range of distances where blur may provide a useful depth cue (approximately $\geq 2 \mathrm{~m}$, (Held et al., 2012). Then, it would be possible to determine if there is a) a co-variance in 
depth and local slope b) if the co-variance can be measured in a perceived blur task.

\subsection{Blur adaptation}

Results from blur adaptation experiments (Webster et al., 2002) are consistent with our data collected in the lab. Recall that observers were allowed to view as many random dead leaves patches as they wished while tracing; observers often choose to view several of distinct dead leaves references patches before completing their tracing which may have lead to some degree of blur adaptation. We did not measure, direct, or control adaptation during the experiment, and a more systematic exploration with more rigorous methods would be required to determine how the adaptation state of the observer influences a blur tracing task. Despite the lack of control, there are hints in the data that blur adaptation may have played a role in our experiment. For example, we observed (see Figs. 6 and 7) that observers traced regions that were sharper/blurry than the standard when it was blurry/sharp. Webster et al. found similar effects, using a global slope measure, in an adaptation task. When observers adapted to blurry images, standard images appeared more blurred than when not adapted, and for adaptation to sharp images the converse was true.

\subsection{Mechanical Turk's suitability for vision research}

Despite the objections noted above, it is worth considering what role Mechanical Turk (and other online data collection systems) may be suited to play in vision research. From the work on cognition (Crump et al., 2013) and other online experiments (Verhallen et al., 2014) it is reasonable to suggest that relatively high-level perceptual tasks may benefit from the ease and speed of data collection Mechanical Turk provides. There is some preliminary work that addresses face perception using reverse-correlation online (Jolij et al., 2014), but direct replication of work done in the lab and online would be required to reveal the quality of the online data or if there are systematic differences that appear from the two samples. However, our data are encouraging in that some tasks, even those with relatively basic 
perception questions, could be addressed with large samples of online participants which could facilitate work on topics such as individual differences (e.g., Baker, 2013; Goodbourn et al., 2012).

\subsection{Conclusion}

The local slope of contrast versus spatial scale provides a computationally simple metric of local image blur and predicts observerś perceived blur in a free tracing task both under controlled lab conditions and in an online experiment.

\section{References}

Baker, D. H., 2013. What is the primary cause of individual differences in contrast sensitivity? PloS one 8 (7), e69536.

Bex, P., Edgar, G., Smith, A., 1995. Sharpening of drifting, blurred images. Vision research 35 (18), 2539-2546.

Bex, P. J., Solomon, S. G., Dakin, S. C., 2009. Contrast sensitivity in natural scenes depends on edge as well as spatial frequency structure. Journal of Vision 9 (10), 1.

Bordenave, C., Gousseau, Y., 2006. The dead leaves model: a general tessellation modeling occlusion. Advances in applied probability.

Brainard, D., 1997. The psychophysics toolbox. Spatial vision 10 (4), 433-436.

Buhrmester, M., Kwang, T., Gosling, S. D., 2011. Amazon's mechanical turk a new source of inexpensive, yet high-quality, data? Perspectives on Psychological Science 6 (1), 3-5.

Burge, J., Geisler, W., 2011. Optimal defocus estimation in individual natural images. Proceedings of the National Academy of Sciences 108 (40), 16849-16854. 
Burr, D., Morgan, M., 1997. Motion deblurring in human vision. Proceedings of the Royal Society of London. Series B: Biological Sciences 264 (1380), 431-436.

Ciuffreda, K. J., Selenow, A., Wang, B., Vasudevan, B., Zikos, G., Ali, S. R., 2006. bothersome blur: A functional unit of blur perception. Vision research 46 (6), 895-901.

Ciuffreda, K. J., Wang, B., Wong, D., 2005. Central and near peripheral retinal contributions to the depth-of-focus using naturalistic stimulation. Vision research 45 (20), 2650-2658.

Commons, C., 2001. Creative commons. Creative Commons.

Crump, M. J., McDonnell, J. V., Gureckis, T. M., 2013. Evaluating amazon's mechanical turk as a tool for experimental behavioral research. PloS one 8 (3), e57410.

DeValois, R., DeValois, K., 1988. Spatial vision. No. 14. Oxford University Press, USA.

Elder, J., 1999. Are edges incomplete? International Journal of Computer Vision 34 (2), $97-122$.

Ens, J., Lawrence, P., 1993. An investigation of methods for determining depth from focus. Pattern Analysis and Machine Intelligence, IEEE Transactions on 15 (2), 97-108.

Field, D. J., Brady, N., et al., 1997. Visual sensitivity, blur and the sources of variability in the amplitude spectra of natural scenes. Vision research 37 (23), 3367-3383.

Field, D. J., et al., 1987. Relations between the statistics of natural images and the response properties of cortical cells. J. Opt. Soc. Am. A 4 (12), 2379-2394.

Freeman, W. T., Adelson, E. H., 1991. The design and use of steerable filters. IEEE Transactions on Pattern analysis and machine intelligence 13 (9), 891-906.

Galvin, S. J., O’Shea, R. P., Squire, A. M., Govan, D. G., 1997. Sharpness overconstancy in peripheral vision. Vision research 37 (15), 2035-2039. 
Georgeson, M., 1985. The effect of spatial adaptation on perceived contrast. Spatial vision $1(2), 103-112$.

Georgeson, M. A., May, K. A., Freeman, T. C., Hesse, G. S., 2007. From filters to features: Scale-space analysis of edge and blur coding in human vision. Journal of vision 7 (13).

Goodbourn, P. T., Bosten, J. M., Hogg, R. E., Bargary, G., Lawrance-Owen, A. J., Mollon, J., 2012. Do different magnocellular tasks probe the same neural substrate? Proceedings of the Royal Society B: Biological Sciences, rspb20121430.

Haun, A. M., Peli, E., 2011. 24.1: Measuring the perceived contrast of natural images. In: SID Symposium Digest of Technical Papers. Vol. 42. Wiley Online Library, pp. 302-304.

Held, R. T., Cooper, E. A., Banks, M. S., 2012. Blur and disparity are complementary cues to depth. Current Biology 22 (5), 426-431.

Hodos, W., Kuenzel, W., 1984. Retinal-image degradation produces ocular enlargement in chicks. Investigative ophthalmology \& visual science 25 (6), 652-659.

Ipeirotis, P. G., 2010. Demographics of mechanical turk.

Jolij, J., Wriedt, S. C., Luckmann, A., 2014. Chronic negative mood affects internal representations of negative facial expressions-an internet study. Tech. rep., PeerJ PrePrints.

Kleiner, M., Brainard, D., Pelli, D., Ingling, A., Murray, R., Broussard, C., 2007. Whats new in psychtoolbox-3. Perception 36, 14.

Lee, A., Mumford, D., 2001. Occlusion models for natural images: A statistical study of a scale-invariant dead leaves model. International Journal of Computer Vision.

Maji, S., 2011. Large scale image annotations on amazon mechanical turk. EECS Department, University of California, Berkeley, Tech. Rep. UCB/EECS-2011-79. 
Mante, V., Frazor, R. A., Bonin, V., Geisler, W. S., Carandini, M., 2005. Independence of luminance and contrast in natural scenes and in the early visual system. Nature neuroscience 8 (12), 1690-1697.

Marziliano, P., Dufaux, F., Winkler, S., Ebrahimi, T., 2002. A no-reference perceptual blur metric. In: Image Processing. 2002. Proceedings. 2002 International Conference on. Vol. 3. IEEE, pp. III-57.

Mather, G., 1997. The use of image blur as a depth cue. PERCEPTION-LONDON- 26, $1147-1158$.

Mather, G., Smith, D., 2000a. Depth cue integration: stereopsis and image blur* 1. Vision Research 40 (25), 3501-3506.

Mather, G., Smith, D. R., 2000b. Depth cue integration: stereopsis and image blur. Vision research 40 (25), 3501-3506.

Mather, G., Smith, D. R., 2002. Blur discrimination and its relation to blur-mediated depth perception. PERCEPTION-LONDON- 31 (10), 1211-1220.

McCormick, E., De Volder, K., 2004. Jquery: finding your way through tangled code. In: Companion to the 19th annual ACM SIGPLAN conference on Object-oriented programming systems, languages, and applications. ACM, pp. 9-10.

McIlhagga, W. H., May, K. A., 2012. Optimal edge filters explain human blur detection. Journal of Vision 12 (10).

Morgan, M. J., Watt, R. J., 1997. The combination of filters in early spatial vision: a retrospective analysis of the mirage model. PERCEPTION-LONDON- 26, 1073-1088.

Murray, S., Bex, P. J., 2010. Frontiers: Perceived blur in naturally contoured images depends on phase. Frontiers in Perception Science 1. 
Olmos, A., Kingdom, F., 2004. A biologically inspired algorithm for the recovery of shading and reflectance images. Perception 33, 1463-1473.

Olzak, L. A., Thomas, J. P., 1999. Neural recoding in human pattern vision: model and mechanisms. Vision Research 39 (2), 231-256.

Pelli, D., 1997. The videotoolbox software for visual psychophysics: transforming numbers into movies. Spatial Vision 10 (4), 437-442.

Pentland, A. P., 1987. A new sense for depth of field. Pattern Analysis and Machine Intelligence, IEEE Transactions on (4), 523-531.

Rashtchian, C., Young, P., Hodosh, M., Hockenmaier, J., 2010. Collecting image annotations using amazon's mechanical turk. In: Proceedings of the NAACL HLT 2010 Workshop on Creating Speech and Language Data with Amazon's Mechanical Turk. Association for Computational Linguistics, pp. 139-147.

Ravikumar, A., Applegate, R. A., Shi, Y., Bedell, H. E., 2011. Six just-noticeable differences in retinal image quality in 1 line of visual acuity: Toward quantification of happy versus unhappy patients with 20/20 acuity. Journal of Cataract \& Refractive Surgery 37 (8), $1523-1529$.

Ross, J., Irani, L., Silberman, M., Zaldivar, A., Tomlinson, B., 2010. Who are the crowdworkers?: shifting demographics in mechanical turk. In: CHI'10 Extended Abstracts on Human Factors in Computing Systems. ACM, pp. 2863-2872.

Saunders, D. R., Bex, P. J., Woods, R. L., 2013. Crowdsourcing a normative natural language dataset: a comparison of amazon mechanical turk and in-lab data collection. Journal of medical Internet research $15(5)$. 
Simmers, A. J., Bex, P. J., Hess, R. F., 2003. Perceived blur in amblyopia. Investigative ophthalmology \& visual science 44 (3), 1395-1400.

Sorokin, A., Forsyth, D., 2008. Utility data annotation with amazon mechanical turk. Urbana $51(61), 820$.

Subbarao, M., Gurumoorthy, N., 1988. Depth recovery from blurred edges. In: Computer Vision and Pattern Recognition, 1988. Proceedings CVPR'88., Computer Society Conference on. IEEE, pp. 498-503.

Taylor, C. P., Bennett, P. J., Sekuler, A. B., 2009. Spatial frequency summation in visual noise. JOSA A 26 (11), B84-B93.

Taylor, C. P., Bennett, P. J., Sekuler, A. B., 2014. Evidence for adjustable bandwidth orientation channels. Perception Science 5, 578.

Tolhurst, D., Tadmor, Y., Chao, T., 1992. Amplitude spectra of natural images. Ophthalmic and Physiological Optics 12 (2), 229-232.

van der Schaaf, v. A., van Hateren, J. v., 1996. Modelling the power spectra of natural images: statistics and information. Vision research 36 (17), 2759-2770.

Verhallen, R. J., Bosten, J. M., Goodbourn, P. T., Bargary, G., Lawrance-Owen, A. J., Mollon, J., 2014. An online version of the mooney face test: phenotypic and genetic associations. Neuropsychologia 63, 19-25.

Vu, C. T., Phan, T. D., Chandler, D. M., 2012. S3: A spectral and spatial measure of local perceived sharpness in natural images. IEEE Transactions on Image Processing 21 (3), 934.

Walker, L., ZuRhein, G., Hodach, A., Chou, S., 1978. Extreme myopia produced by modest change in early visual experience. Science 201, 29. 
Wang, Z., Simoncelli, E., 2003. Local phase coherence and the perception of blur. Adv. Neural Information Processing Systems (NIPS03 16.

Watson, A., Ahumada, A., 2010. Blur clarified. Journal of Vision 10 (7), 1385.

Watt, R., Morgan, M., 1983. The recognition and representation of edge blur: evidence for spatial primitives in human vision. Vision Research 23 (12), 1465-1477.

Watt, S., Akeley, K., Ernst, M., Banks, M., 2005. Focus cues affect perceived depth. Journal of Vision 5 (10).

Webster, M., Georgeson, M., Webster, S., et al., 2002. Neural adjustments to image blur. Nature Neuroscience 5 (9), 839-840.

Webster, M. A., Mizokami, Y., Svec, L. A., Elliott, S. L., 2006. Neural adjustments to chromatic blur. Spatial vision 19 (2), 111-132.

Webster, S. M., Webster, M. A., Taylor, J., Jaikumar, J., Verma, R., 2001. Simultaneous blur contrast. In: Photonics West 2001-Electronic Imaging. International Society for Optics and Photonics, pp. 414-422. 